\title{
Régénération Naturelle Assistée Du Teck (Tectona Grandis L. F.) Dans La Forêt Classée De La Lama Au Bénin
}

\author{
Denis Gnanguenon-Guesse, \\ Kourouma Koura, \\ Sunday Berlioz Kakpo, \\ Alain Jaurès Gbètoho, \\ Augustin Kossi N. Aoudji,
}

Laboratoire des Sciences Forestières (LSF), Faculté des Sciences, Agronomiques (FSA), Université d'Abomey-Calavi (UAC), Calavi Bénin, République du Bénin

\section{Dominique Louppe,}

Centre de coopération Internationale en Recherche Agronomique pour le Développement (CIRAD)

\section{Jean Cossi Ganglo,}

Laboratoire des Sciences Forestières (LSF), Faculté des Sciences, Agronomiques (FSA), Université d'Abomey-Calavi (UAC), Calavi Bénin, République du Bénin

Doi:10.19044/esj.2019.v15n36p414 URL:http://dx.doi.org/10.19044/esj.2019.v15n36p414

\section{Résumé}

La régénération naturelle assistée, technique utilisée pour favoriser le développement du potentiel adventif et du potentiel séminal des arbres, est un mode de reproduction adopté de nos jours pour renouveler et pérenniser les formations forestières. La présente étude a été réalisée dans les plantations de la forêt classée de la Lama, situées entre $6^{\circ} 55^{\prime}$ et $7^{\circ} 00^{\prime}$ de latitude Nord et entre $2^{\circ} 4^{\prime}$ et $2^{\circ} 12^{\prime}$ de longitude Est. Elle vise à proposer une technique de régénération naturelle assistée peu coûteuse adaptée aux peuplements de teck installés sur des vertisols hydromorphes. Trois méthodes de régénérations naturelles assistées ont été testées sur quatre types de sols (vertisol argilosableux, vertisol argileux, vertisol argilo-calcaire et vertisols argilo-calcaire superficiel). Pour chaque traitement sur chaque type de sol, deux placeaux carrées de $100 \mathrm{~m}^{2}$ ont été matérialisés avec chacun huit placettes de $4 \mathrm{~m}^{2}$. La régénération a été inventoriée dans chaque placette et des relevés ont été effectués par rapport au nombre, hauteur et circonférence au collet des jeunes plants. L'évaluation des coûts de la méthode a été réalisée grâce à une 
comptabilité analytique régulière prenant en compte toutes les dépenses d'installation et de maintenance des parcelles expérimentales.

La densité, hauteur et circonférence au collet varient significativement en fonction des types de sol. Toutefois, les plants sont plus développés en hauteur et en circonférence au collet dans les interlignes que sur les lignes. La régénération sur sol non travaillé est le moins coûteuse et donc celui à recommander aux gestionnaires des teckeraies de la Lama.

Mots clés : Bénin, Régénération naturelle assistée, Tectona grandis

\title{
Assisted Natural Regeneration Of Teak (Tectona Grandis L. F.) In Lama Forest Reserve (Benin)
}

\section{Denis Gnanguenon-Guesse, Kourouma Koura, Sunday Berlioz, Kakpo, Alain Jaurès Gbètoho, Augustin Kossi N. Aoudji,}

Laboratoire des Sciences Forestières (LSF), Faculté des Sciences, Agronomiques (FSA), Université d'Abomey-Calavi (UAC), Calavi Bénin, République du Bénin

\section{Dominique Louppe,}

Centre de coopération Internationale en Recherche Agronomique pour le

Développement (CIRAD)

\section{Jean Cossi Ganglo,}

Laboratoire des Sciences Forestières (LSF), Faculté des Sciences, Agronomiques (FSA), Université d'Abomey-Calavi (UAC), Calavi Bénin, République du Bénin

\begin{abstract}
Assisted natural regeneration is a technique used to promote the development of the seminal potential of trees. Actually, it is a mode of reproduction adopted to renew and sustain the forests. This study was carried out in the plantations of the Lama reserve (between $6^{\circ} 55^{\prime}$ and $7^{\circ} 00^{\prime}$ north and between $2^{\circ} 4^{\prime}$ and $2^{\circ} 12^{\prime}$ east). It aims to propose an inexpensive assisted natural regeneration method adapted to teak stands installed on hydromorphic vertisols. Three assisted natural regeneration methods were tested on four soil
\end{abstract}


types ((clay-sandy vertisol, clay vertisol, clay-limestone vertisol, and superficial clay-limestone vertisols). For each treatment about each type of soil, two square plots $\left(100 \mathrm{~m}^{2}\right)$ were materialized with eight plots of $4 \mathrm{~m}^{2}$ each one. Regeneration was inventoried in each plot and data are collected about number, height, and circumference at the neck of seeding. The production costs were assessed through regular cost accounting taking into account all the installation and maintenance expenses of the experimental plots. From the results, density, height, and collar circumference vary significantly with soil types. However, the seedlings are more developed in height and circumference at the collar in the interlinings than on the lines. Regeneration on unworked soil is the least expensive and therefore the one to recommend to managers of teak plantations of the Lama.

Keywords: Benin, Natural assisted regeneration, Tectona grandis

\section{Introduction}

La forêt est une des principales richesses de nombreux pays de la zone intertropicale humide mais elle est en train de régresser face à l'augmentation de la demande en bois et en terres liées à la pression démographique et à l'agriculture industrielle (Putz \& Redford 2010 ; Gnanguenon-Guessè et al., 2015 ; FAO, 2016 ; Kakpo et al., 2018). Ainsi, le Bénin perd environ 50000 hectares de couverture forestière chaque année (FAO, 2010). Pourtant d'après le rapport de la FAO (2014), la valeur ajoutée du secteur forestier serait de 145 millions de US\$ par an, sans que soient comptabilisés le commerce informel $\mathrm{du}$ bois de feu, ni celui de produits forestiers non ligneux, ni l'autoconsommation du bois en milieu rural et urbain.

Dès la fin des années 1940, des actions de reboisement ont été menées pour suppléer à l'insuffisance, déjà prévisible, des ressources forestières naturelles et pour satisfaire les besoins des populations (Verhaegen et al., 2010). Aujourd'hui, les plantations ont aussi comme objectif de freiner la perte des couverts forestiers. Elles visent à produire du bois d'œuvre de qualité. Le teck (Tectona grandis L. f) est, au Bénin, l'une des espèces retenues à cette fin. C'est une espèce caducifoliée de pleine lumière, pionnière à longue durée de vie. Sa phénologie dépend de la zone climatique. Il peut croître sur de nombreux types de sols sauf ceux à engorgement temporaire (de longue durée) ou permanent. Cependant, le teck reste une espèce très exigeante qui, pour une bonne croissance, demande un sol profond, bien drainé, à forte teneur en bases, surtout $\mathrm{Ca}, \mathrm{Mg}$ et $\mathrm{P}$, et un $\mathrm{pH}$ neutre ou légèrement acide (Louppe, 2005).

Le teck a été introduit au Bénin dès 1916 par la mission catholique (Behaghel, 1999) et ses plantations couvrent une superficie de 40000 ha dont 20000 ha de plantations domaniales. Pour renouveler ces peuplements de teck après exploitation, l'Office National du Bois (ONAB) utilise depuis 1989, sur les 
sols ferralitiques et ferrugineux, la technique de régénération naturelle assistée (RNA) (Lutz, 1991 ; Akouèhou \& Ayélo, 2005). La régénération naturelle est un élément de la dynamique des écosystèmes forestiers tropicaux. Elle peut être utilisée pour orienter la reconstitution des forêts et définir les possibilités réelles d'aménagement dans le domaine des plantations forestières (Marien \& Mallet, 2004). La régénération naturelle assistée consiste à créer des conditions favorables au développement spontané du potentiel adventif et du potentiel séminal y compris édaphique en dormance (Marien \& Mallet, 2004 ; Akouèhou \& Ayélo, 2005) ou, selon la FAO (2005), à protéger des plants d'espèces à valeur économique, sociale et culturelle reconnue et à améliorer leur croissance.

Il existe plusieurs méthodes pour multiplier les végétaux, en fonction du type de plantes. Généralement le forestier planteur utilise la reproduction sexuée ou la multiplication végétative pour produire, en pépinière des plants, qui seront transportés et mis en terre sur le terrain. Plus rarement, il sème des graines directement sur le terrain pour éviter les coûts de pépinière. La régénération naturelle assistée est une autre voie qui vise à améliorer le renouvellement naturel et spontané des forêts.

La régénération naturelle des peuplements est moins coûteuse que la plantation. Elle procure une stabilité et une adaptation plus adéquates à l'environnement (Lutz, 1991). Par contre, on note un appauvrissement du stock génétique au fil des années car le mode de propagation par graines présente plusieurs limites (Monteuuis et al., 2007 ; Kjaer et al. 2000 ; Akpenè et al., 2014).

Au Bénin, la technique de régénération naturelle assistée consiste à diviser la parcelle à régénérer en bandes parallèles de 30 mètres de large. Une bande sur deux est exploitée à blanc la première année. La seconde est conservée pour le maintien du système écologique existant afin d'éviter l'érosion hydrique du sol. Le bois d'œuvre et de service est vendu aux industriels. Les branchages sont ramassés par les populations riveraines avant le démarrage de la saison pluvieuse. Au début de la saison des pluies suivante, la végétation adventice est fauchée, les feuilles mortes et les brindilles sont mises en tas et brûlées pour laisser un terrain propre où les graines vont pouvoir germer. On obtient ainsi de 7000 à 20000 semis par hectare. La bande conservée est exploitée l'année suivante. Cette technique est bien adaptée au teck poussant sur des sols filtrants (ferralitiques et ferrugineux). Cette technique est -elle transposable aux vertisols hydromorphes de LAMA ? Ces sols mal drainés, où les inondations sont fréquentes, ne seraient-ils pas un handicap pour la régénération naturelle d'une espèce exigeante comme le teck ? 


\section{Matériel et méthodes}

\section{Milieu d'étude}

Les plantations de teck étudiées sont situées dans la forêt classée de la Lama, entre $6^{\circ} 51^{\prime}$ et $7^{\circ} 04^{\prime}$ de latitude nord et $2^{\circ} 03^{\prime}$ et $2^{\circ} 22^{\prime}$ de longitude est, dans les secteurs forestiers de Massi, de Koto et d'Akpè (Figure 1). Elles couvrent une superficie de 7200 ha. C'est une zone de transition entre le climat subéquatorial et le climat tropical où la pluviosité moyenne annuelle varie de $1100 \mathrm{~mm}$ à $1200 \mathrm{~mm}$. Il y a quatre saisons : une grande saison de pluies de mars à juillet, une petite saison sèche en août, une petite saison de pluies de septembre à octobre et la grande saison sèche de novembre à février.
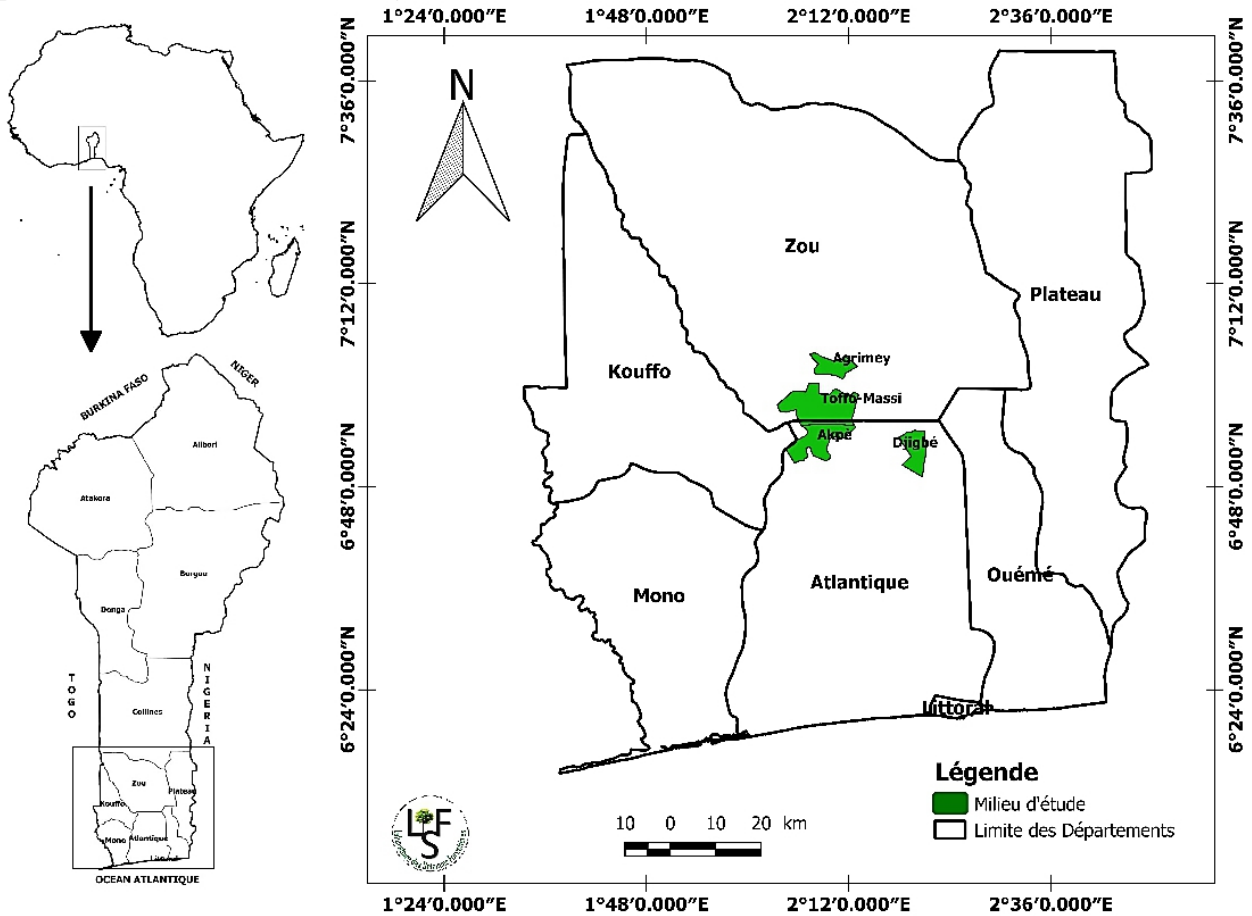

Figure 1: Localisation de la forêt de Lama (source : Gnanguènon-Guéssè, 2019)

\section{Paramètres dendrométriques des régénérations} Sites d'expérimentation

Les études sur les possibilités d'utiliser la RNA sur les vertisols ont été menées dans quatre parcelles forestières $(6 \mathrm{a}, 10 \mathrm{a}, 17 \mathrm{a}$ et $25 \mathrm{a})$, qui devaient être régénérées par semis naturel en 2017. Chacune de ces parcelles présente un type de sol différent : la parcelle 6a de Massi est sur un sol argileux à recouvrement sableux (AS), la parcelle 25a de Massi sur un vertisol argileux (VA), la parcelle 17a dans le secteur d'Akpè sur un vertisol argilo-calcaire superficiel (VACS) et la parcelle 10a de Koto sur un vertisol argilo-calcaire (VAC) (Viennot, 1966). 


\section{Dispositif expérimental}

Le dispositif expérimental mis en place a pour but de comparer le système usuel de régénération naturelle assistée décrit ci-dessus avec la régénération par semis de graines sur billons et sur buttes qui a pour objectif d'éviter la submersion des graines et l'asphyxie des racines des jeunes plants pendant la saison des pluies.

C'est un essai en blocs complets à quatre répétitions dans lequel chaque bloc correspond à un des types de sols décrits ci-dessus. Chaque bloc, compte trois parcelles d'essai orientées nord-sud, de $30 \times 70 \mathrm{~m}$ soit 0,21 ha séparées par une bande non exploitée (Figure 2). Dans chacune de ces parcelles, les arbres ont été abattus puis débardés. Les trois traitements ont été disposés de manière systématique :

- À l'ouest, le témoin a consisté en l'élimination des rémanents, suivi d'un désherbage pour laisser un sol nu non travaillé.

- La parcelle du second traitement, au centre, a été billonné à la houe après dégagement du terrain. Les billons ont une largeur moyenne de $1 \mathrm{~m}$ et une hauteur de $50 \mathrm{~cm}$.

- Dans le troisième traitement, à l'est, le travail du sol a consisté en un buttage à la houe. Les buttes ont un diamètre de $60 \mathrm{~cm}$ à la base et une hauteur de $50 \mathrm{~cm}$.

Les billons et les buttes sont faits sur les anciennes lignes de plantation. Des graines de teck ont ensuite été semées sur les billons et les buttes.

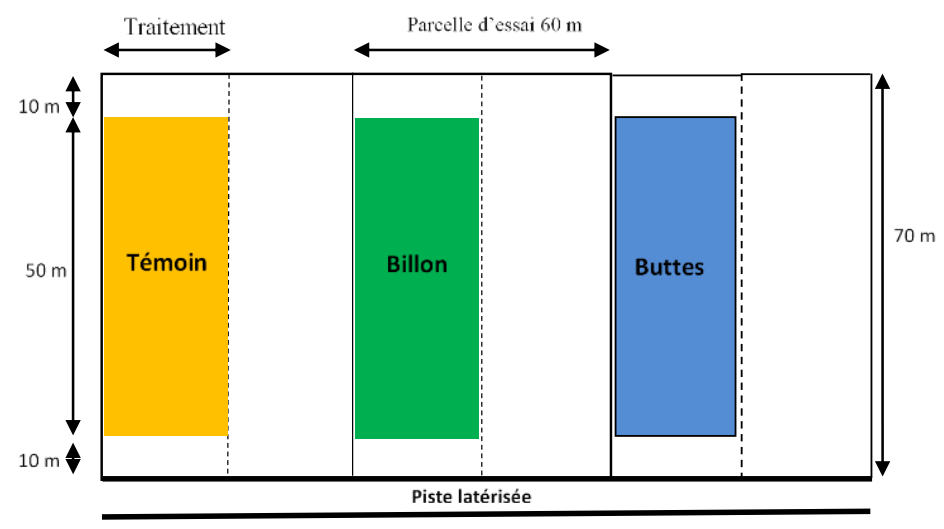

Figure 2 : Disposition des parcelles d'essai expérimentales (source : Gnanguènon-Guéssè, 2019)

Tous les traitements ont, une fois par mois pendant six mois, été désherbés et débarrassés des rejets de souches pour éviter la concurrence. Dans chacune des parcelles ont été installés deux placeaux de 10 mètres de côté (un are), séparés de $10 \mathrm{~m}$, tout en évitant les micro-cuvettes qui risqueraient de biaiser les résultats. Pour limiter les effets de bordure et les effets d'ombrage, les placeaux sont à $20 \mathrm{~m}$ de la piste d'accès et la lisière terminales et à $10 \mathrm{~m}$ 
des lisières latérales (figure 3). Les placeaux dans lesquels sont effectués les comptages de germination et la mesure des plantules comportent trois lignes de plantation et deux interlignes.

Lisière terminale

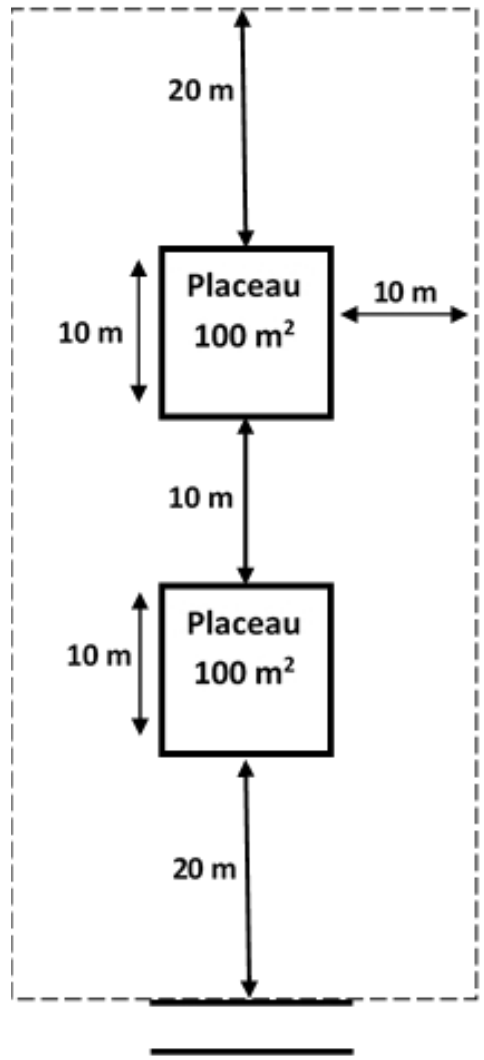

Piste latérisée

Figure 3 : Disposition des placeaux de mesure dans les parcelles expérimentales (source : Gnanguènon-Guéssè, 2019)

Dans chacun des placeaux, dix (10) placettes de $4 \mathrm{~m}^{2}$ ont été matérialisées : 6 placettes rectangulaires $(4 \mathrm{mx} 1 \mathrm{~m})$ sur lignes de plantation où sont situés les billons et les buttes et 4 placettes carrées $(2 \mathrm{~m} \times 2 \mathrm{~m})$ dans les interlignes (figure 4). 


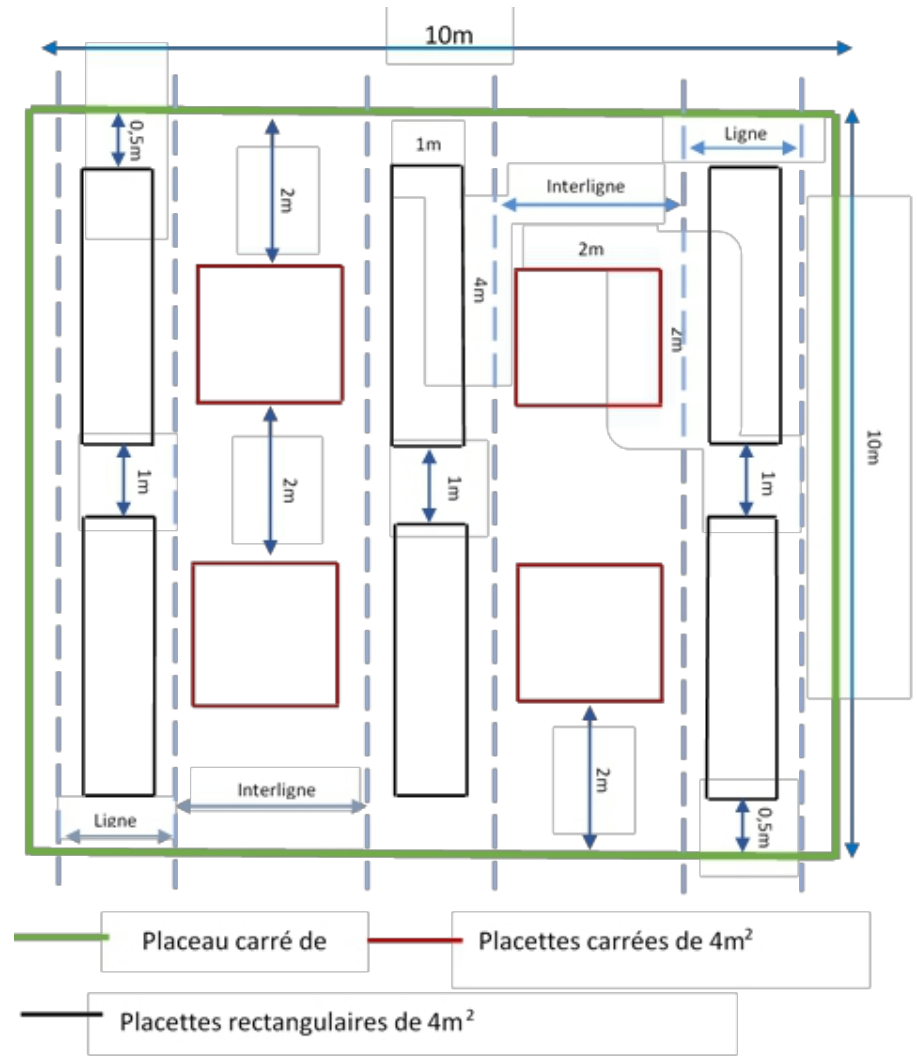

Figure 4 : Schéma des placettes dans le placeau du témoin et des billons (source : Gnanguènon-Guéssè, 2019)

Dans chacun des placeaux où le travail du sol consiste à façonner des buttes, 4 placettes carrées $(2 \mathrm{mx} 2 \mathrm{~m})$ ont été installées dans les interlignes et sur les lignes toutes les buttes ont été considérées comme des unités d'échantillonnage (figure 5). 


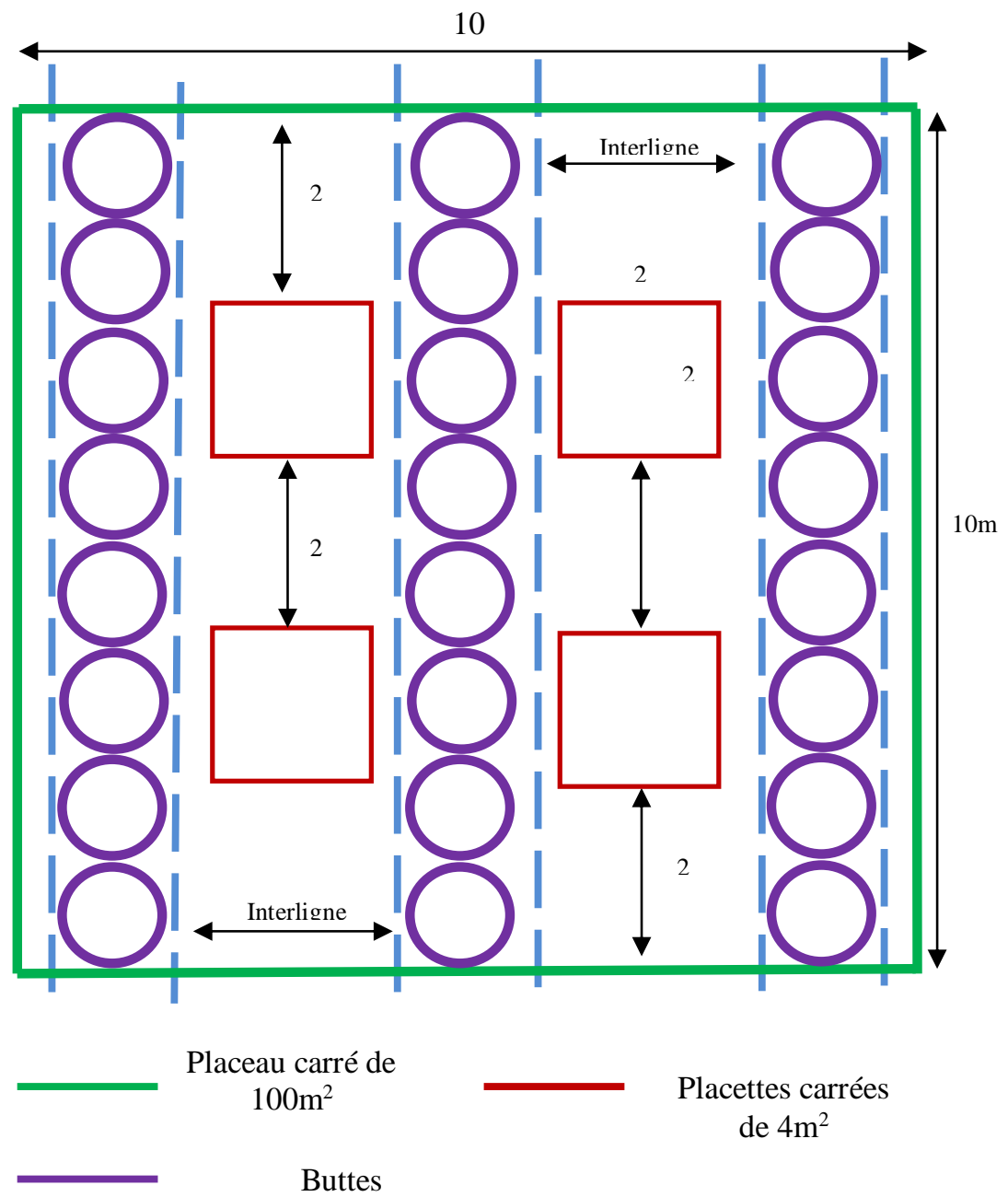

Figure 5 : Schéma des placettes dans le placeau des buttes (source : Gnanguènon-Guéssè, 2019)

\section{Collecte des données}

Dans chaque placette et sur chaque butte, on a procédé au comptage systématique des plants par classe de hauteurs $(\mathrm{Ht}<0,5 \mathrm{~m} ; 0,5 \leq \mathrm{Ht}<1 \mathrm{~m}$ et $\mathrm{Ht}$ $\geq 1 \mathrm{~m}$ ). Les mesures de la hauteur ont été prises avec une perche graduée et celles de la circonférence avec un mètre de couturier. La circonférence au collet et la hauteur ont été mesurées sur le premier de chaque série de quatre plants dans le sens de la progression

\section{Traitement et analyse des données}

Les données d'inventaire sont saisies à l'aide du logiciel Excel. Les principaux paramètres dendrométriques suivants sont calculés.

La densité à l'hectare $\left(\mathrm{N}_{\mathrm{i}}\right)$ de chaque surface échantillonnée : 


$$
N_{i}=\frac{N e}{S} \times 10000
$$

avec Ne le nombre de plants par butte ou par placette et $\mathrm{S}$ la surface de la section d'une butte $S=\left(\frac{0,60}{2}\right)^{2} \times \pi=0,2826 \mathrm{~m}^{2}$ ou d'une placette $\mathrm{S}=4 \mathrm{~m}^{2}$.

La densité moyenne $(\mathrm{N})$ de plants par sous-bande du placeau :

$$
N=\frac{\sum d_{i}}{n}
$$

avec $\mathrm{n}$ le nombre total de buttes ou de placettes par emplacement (sommet ou interligne) par placeau.

La hauteur moyenne (Hm, en $\mathrm{m})$. Elle est la hauteur moyenne des plants échantillons par placeau.

$$
\mathrm{H}_{m}=\frac{\sum_{\mathrm{i}=1}^{\mathrm{n}} \mathrm{h}_{\mathrm{i}}}{n}
$$

Avec $\mathrm{Hm}=$ hauteur moyenne des plants, hi $=$ la hauteur du plant $\mathrm{i}$ échantillonné et $\mathrm{n}=$ le nombre de plants échantillonnés par placette ou par butte.

La circonférence moyenne au collet $(\mathrm{Ccm}$, en $\mathrm{cm})$.

$$
C c m=\frac{\sum_{\mathrm{i}=1}^{\mathrm{n}} c_{\mathrm{i}}}{n}
$$

Avec $\mathrm{C}$ la circonférence moyenne au collet des plants, $\mathrm{ci}=\mathrm{la}$ circonférence au collet de chaque plant mesuré et $\mathrm{n}=$ le nombre de plants mesurés par placette ou par butte.

Après vérification de la normalité et de l'homogénéité des variances, le test $t$ de Student a été utilisé pour comparer les modes de préparation du sol par type de sol en comparant les densités moyennes de plants, les hauteurs moyennes et les circonférences moyennes, ceci sur les lignes et dans les interlignes.

Des analyses de variance à deux facteurs ont été utilisées pour définir l'impact du type de sol et du mode de préparation du sol, et leurs interactions. Avant la réalisation de l'analyse de variance, la normalité a été vérifiée grâce à un test de Shapiro-Wilk. L'égalité des variances inter-bloc et inter-mode de préparation du sol a été vérifiée grâce au test de Levene. Suite à l'analyse de variance et la vérification de l'absence d'interaction grâce à un test d'additivité, un test de Student-Newman-Keuls (SNK) est effectué lorsque qu'un facteur (sol ou traitement) influence significativement un paramètre dendrométrique. Ce test est une procédure de comparaisons multiples deux à deux des moyennes des modalités des facteurs d'intérêts qui sont significativement différente dans l'ANOVA. 


\section{Evaluation des coûts liés à la préparation du sol}

Les dépenses comptabilisées concernent la mise en place de parcelles de $1500 \mathrm{~m}^{2}$ (abattage, nettoyage, billonnage et buttage), des placeaux et des placettes, les désherbages et l'élimination des rejets de souche.. Après l'évacuation des grumes, le dégagement des parterres de coupe a été réalisé par les populations riveraines qui récupère et commercialise les rémanents ; cela n'a entrainé aucun coût supplémentaire.

Pour le traitement témoin, les coûts se sont limités à cinq entretiens à raison de 18.000 FCFA (Franc de la Communauté Financière Africaine) chacun.

Concernant le billonnage : il y a 7 billons par parcelle de $1500 \mathrm{~m}^{2}$ dont le façonnage coûte 4.000 FCFA par billon, soit 28.000 FCFA par parcelle. Il y a également eu cinq (05) entretiens à raison de 18.000 FCFA l'entretien.

Concernant le buttage, le coût d'une butte est de 100 FCFA. Avec 270 buttes pour un parcelle de $1500 \mathrm{~m}^{2}$, le coût d'installation est de 27.000 FCFA par parcelle auxquels s'ajoutent les coûts des cinq entretiens.

Le prix d'achat des graines de teck (descendance d'une provenance tanzanienne récoltée localement) est de 300 FCFA le $\mathrm{kg}$ ( 2 sacs de $40 \mathrm{~kg}$ à l'hectare à raison de $300 \mathrm{FCFA}$ le kg) est de $24.000 \mathrm{FCFA}$; celui de l'épandage des graines $(1 \mathrm{~h} / \mathrm{j}$ par sac de $40 \mathrm{Kg})$ est de $2.520 \mathrm{FCFA}$ et celui des entretiens est de 18.000 FCFA le tour. Le coût de maintenance des ouvriers est également estimé à 10\% des coûts des autres dépenses par traitement.

\section{Résultats}

\section{Caractéristiques des régénérations par traitement et par type de sol}

Le tableau 3 ci-dessous présente les caractéristiques dendrométriques des régénérations de teck obtenues, à savoir: la densité par classe de hauteur, la densité totale, la hauteur moyenne et la circonférence au collet des plants par traitement, par type de sol et selon les emplacements (ligne et interligne). 


\begin{tabular}{|c|c|c|c|c|c|c|c|c|}
\hline \multirow[t]{2}{*}{ Sol } & \multirow{2}{*}{ Traitement } & \multirow[t]{2}{*}{ Emplacement } & \multicolumn{3}{|c|}{ Nombre de plants/ha } & \multirow{2}{*}{$\begin{array}{c}\text { Nombre total de } \\
\text { plants/ha }\end{array}$} & \multirow{2}{*}{$\begin{array}{l}\text { Hauteur } \\
(\mathbf{c m})\end{array}$} & \multirow{2}{*}{$\begin{array}{c}\text { Circonférence } \\
(\mathbf{m m})\end{array}$} \\
\hline & & & Ht $<0,5 \mathrm{~m}$ & $0,5 \leq \mathrm{Ht}<1 \mathrm{~m}$ & $\mathbf{H t} \geq \mathbf{1 ~ m}$ & & & \\
\hline \multirow{6}{*}{$\begin{array}{c}\text { Vertisol } \\
\text { Argileux à } \\
\text { recouvrement } \\
\text { sableux (AS) }\end{array}$} & \multirow[t]{2}{*}{ Témoin } & Interligne & $12187 \pm 3094$ & $2812 \pm 3977$ & $312 \pm 442$ & $15312 \pm 7513$ & $33 \pm 25$ & $34 \pm 0,2$ \\
\hline & & Ligne & $13333 \pm 0$ & $208 \pm 295$ & 0 & $13542 \pm 295$ & $29 \pm 5$ & $25 \pm 1,5$ \\
\hline & \multirow[t]{2}{*}{ Billon } & Interligne & $12812 \pm 442$ & $2500 \pm 884$ & 0 & $15312 \pm 1326$ & $42 \pm 14$ & $55 \pm 12,3$ \\
\hline & & Ligne & $25000 \pm 5893$ & 0 & 0 & $25000 \pm 5893$ & $21 \pm 4$ & $27 \pm 1,2$ \\
\hline & \multirow[t]{2}{*}{ Butte } & Interligne & 26563 & 0 & 0 & 26563 & $21 \pm 2$ & $30 \pm 1,6$ \\
\hline & & Ligne & $23590 \pm 10009$ & 0 & 0 & $23590 \pm 10009$ & $15 \pm 2$ & $22 \pm 8,1$ \\
\hline \multirow{6}{*}{$\begin{array}{l}\text { Vertisol } \\
\text { Argileux } \\
\text { (VA) }\end{array}$} & \multirow[t]{2}{*}{ Témoin } & Interligne & $23437 \pm 6629$ & $6562 \pm 442$ & $2812 \pm 1326$ & $32812 \pm 4861$ & $58 \pm 17$ & $63 \pm 11$ \\
\hline & & Ligne & $26667 \pm 15910$ & $8333 \pm 0$ & $1458 \pm 295$ & $36458 \pm 16205$ & $54 \pm 1$ & $62 \pm 6,5$ \\
\hline & \multirow[t]{2}{*}{ Billon } & Interligne & $18437 \pm 7513$ & $6875 \pm 2652$ & $6562 \pm 7513$ & $31875 \pm 2652$ & $72 \pm 38$ & $71 \pm 8,1$ \\
\hline & & Ligne & $29583 \pm 17088$ & $13542 \pm 5598$ & $5208 \pm 5009$ & $48333 \pm 17678$ & $67 \pm 35$ & $62 \pm 23,3$ \\
\hline & \multirow[t]{2}{*}{ Butte } & Interligne & 16875 & 10313 & 3750 & 30938 & $73 \pm 2$ & $65 \pm 1,1$ \\
\hline & & Ligne & $52732 \pm 7850$ & $5898 \pm 8340$ & $2949 \pm 4170$ & $61578 \pm 4661$ & $31 \pm 18$ & $40 \pm 10,3$ \\
\hline \multirow{6}{*}{$\begin{array}{c}\text { Vertisol } \\
\text { Argilo- } \\
\text { Calcaire } \\
\text { (VAC) }\end{array}$} & \multirow[t]{2}{*}{ Témoin } & Interligne & $28437 \pm 3094$ & $625 \pm 0$ & 0 & $29062 \pm 3094$ & $28 \pm 7$ & $44 \pm 5$ \\
\hline & & Ligne & $31667 \pm 1179$ & $208 \pm 295$ & 0 & $31875 \pm 1473$ & $24 \pm 01$ & $42 \pm 0,4$ \\
\hline & \multirow[t]{2}{*}{ Billon } & Interligne & $12500 \pm 15026$ & $625 \pm 884$ & 0 & $13125 \pm 15910$ & $24 \pm 11$ & $39 \pm 13$ \\
\hline & & Ligne & $47500 \pm 32409$ & $1042 \pm 1473$ & 0 & $48542 \pm 33882$ & $24 \pm 5$ & $33 \pm 8$ \\
\hline & \multirow[t]{2}{*}{ Butte } & Interligne & 34688 & 0 & 0 & 34688 & $26 \pm 0,3$ & $43 \pm 10,7$ \\
\hline & & Ligne & $113442 \pm 30909$ & $2082 \pm 2944$ & 0 & $115524 \pm 33853$ & $25 \pm 0,1$ & $46 \pm 1,1$ \\
\hline \multirow{6}{*}{$\begin{array}{c}\text { Vertisol } \\
\text { Argilo- } \\
\text { Calcaire } \\
\text { superficiel } \\
\text { (VACS) }\end{array}$} & \multirow[t]{2}{*}{ Témoin } & Interligne & $30625 \pm 13258$ & $1562 \pm 442$ & 0 & $32187 \pm 13700$ & $33 \pm 1$ & $51 \pm 2,5$ \\
\hline & & Ligne & $38958 \pm 2062$ & $9583 \pm 7071$ & $208 \pm 295$ & $48750 \pm 8839$ & $34 \pm 3$ & $43 \pm 0,5$ \\
\hline & \multirow[t]{2}{*}{ Billon } & Interligne & $22812 \pm 145847$ & $5625 \pm 7955$ & $625 \pm 884$ & $29062 \pm 23423$ & $30 \pm 6$ & $37 \pm 0,8$ \\
\hline & & Ligne & $37292 \pm 3830$ & $2083 \pm 589$ & $833+0$ & $40208,33 \pm 4419,41$ & $28 \pm 2$ & $35 \pm 1,9$ \\
\hline & \multirow[t]{2}{*}{ Butte } & Interligne & 9063 & 3125 & 1563 & 13751 & $58 \pm 2$ & $65 \pm 1,7$ \\
\hline & & Ligne & $7274 \pm 3614$ & $983 \pm 1390$ & 0 & $8257 \pm 5004$ & $33 \pm 15$ & $66 \pm 23$ \\
\hline
\end{tabular}

Tableau 3: Description des régénérations de teck obtenues 


\section{Densité moyenne de régénération naturelle assistée}

Le tableau 3, montre que quel que soit le traitement, la régénération obtenue est largement suffisante pour reconstituer un peuplement dense de teck avec plus de 3000 plants à l'hectare dans le pire des cas (8 257 plants \pm 5004 sur les buttes du sol argilo-calcaire superficiel). Considérant le facteur traitement (témoin, butte et billon), il ressort des résultats qu'au niveau des témoins, les densités varient de 15313 à 32813 plants/ha dans les interlignes et de 13542 à 48750 plants/ha sur les lignes avec la plus forte densité (48 750 plants/ha) sur le vertisol argileux calcaire à faible profondeur et la plus faible (13 542 plants/ha) sur le sol argileux sableux. Sur les billons les densités varient de 13125 à 31875 plants/ha dans les interlignes et de 25000 à 48542 plants/ha sur les lignes avec la plus forte densité (48 542) sur le vertisol argileux calcaire et la plus faible (13 125) sur le même vertisol. Sur les buttes, elles varient de 13750 à 34688 plants/ha dans les interlignes et de 8257 à 61 578 plants/ha sur les lignes avec la plus forte densité (61 578) sur le vertisol argileux et la plus faible (8 257) sur le vertisol argileux calcaire à faible profondeur.

Pour ce qui concerne le facteur sol, sur le sol argileux sableux, les densités varient de 15313 à 26563 plants/ha dans les interlignes et de 13542 à 25000 plants/ha sur les lignes avec la plus forte densité (26 563 plants/ha) sur les buttes et la plus faible (13 542 plants/ha) sur le traitement témoin. Sur le vertisol argileux, les densités varient de 30938 à 32813 plants/ha dans les interlignes et de 36458 à 61578 plants/ha sur les lignes avec la plus forte (61 578 plants/ha) et la plus faible (30 938 plants/ha) densité sur les buttes. Sur le vertisol argileux calcaire, elles varient de 13125 à 34688 plants/ha dans les interlignes et de 11552 à 48542 plants/ha sur les lignes avec la plus forte densité (48 542 plants/ha) sur les billons et la plus faible (11 552 plants/ha) sur les buttes. Sur le vertisol argileux calcaire à faible profondeur, la densité varie de 13750 à 32188 plants/ha dans les interlignes et de 8257 à 48750 plants à l'hectare sur les lignes avec une plus forte densité (48 750 plants/ha sur le traitement témoin et la plus faible sur les buttes.

L'analyse de variance (tableau 4) montre que le nombre total de plants diffère significativement selon les types de sol $(\mathrm{p}=0,028)$ mais ne varie pas significativement suivant le traitement $(\mathrm{p}=0,722)$. Ce qui montre l'influence du sol sur le taux de germination. Il faut noter que les caractéristiques physicochimiques des sols sont très variables. Le test d'additivité de Tukey renseigne qu'aucune interaction n'est observée entre le type de sol et le traitement $(\mathrm{p}=0,391)$. La détermination des différences existant entre les traitements sur la base du test de Student-Newman-Keuls montre que les types de sol vertisol argileux et vertisol argilo-calcaire constituent un groupe significativement différent du groupe constitué par le vertisol argilo-calcaire superficiel et le vertisol argileux à recouvrement sableux. 


\section{Hauteur moyenne des régénérations naturelles assistées}

Pour les traitements, il ressort des résultats au niveau des cas témoin que la hauteur des plants varie de $0,28 \mathrm{~m}$ à $0,58 \mathrm{~m}$ dans les interlignes et 0,24 $\mathrm{m}$ à $0,54 \mathrm{~m}$ sur les lignes avec une forte croissance $(0,58 \mathrm{~m})$ sur le vertisol argileux et une plus faible $(0,24 \mathrm{~m})$ sur le vertisol argileux calcaire. Sur les billons, la hauteur enregistrée varie de 0,24 à $0,72 \mathrm{~m}$ dans les interlignes et de $0,22 \mathrm{~m}$ à $0,67 \mathrm{~m}$ sur les lignes avec une forte croissance $(0,72 \mathrm{~m})$ sur le vertisol argileux et une plus faible $(0,22 \mathrm{~m})$ sur le sol argileux sableux. Sur les buttes, elle varie $0,21 \mathrm{~m}$ à $0,73 \mathrm{~m}$ dans les interlignes et de $0,15 \mathrm{~m}$ à $0,33 \mathrm{~m}$ sur les lignes avec une forte croissance $(0,73 \mathrm{~m})$ sur le vertisol argileux et une plus faible $(0,15 \mathrm{~m})$ sur le sol argileux sableux.

Par rapport au facteur sol, sur le sol argileux sableux, la hauteur des plants varie de $0,21 \mathrm{~m}$ à $0,42 \mathrm{~m}$ dans les interlignes et de $0,15 \mathrm{~m}$ à $0,29 \mathrm{~m}$ sur les lignes avec une forte croissance $(0,42 \mathrm{~m})$ sur le traitement des billons et une faible $(0,15 \mathrm{~m})$ au niveau des buttes. Sur le vertisol argileux, la hauteur varie $0,58 \mathrm{~m}$ à $0,73 \mathrm{~m}$ dans les interlignes et de $0,31 \mathrm{~m}$ à $0,67 \mathrm{~m}$ sur les lignes avec de forte croissance sur les traitements des buttes $(0,73 \mathrm{~m})$ et des billons $(0,72 \mathrm{~m})$ et de faible $(0,31 \mathrm{~m})$ au niveau des buttes. Sur le vertisol argileux calcaire, elle varie de $0,24 \mathrm{~m}$ à $0,28 \mathrm{~m}$ dans les interlignes et de $0,24 \mathrm{~m}$ à 0,25 $\mathrm{m}$ sur les lignes avec une forte croissance $(0,28 \mathrm{~m})$ sur le traitement témoin et une faible $(0,24 \mathrm{~m})$ au niveau des traitements témoin et billon. Sur le vertisol argileux calcaire à faible profondeur, elle varie de $0,30 \mathrm{~m}$ à $0,58 \mathrm{~m}$ dans les interlignes et de $0,28 \mathrm{~m}$ à $0,34 \mathrm{~m}$ au niveau des lignes avec une forte croissance $(0,58 \mathrm{~m})$ sur les buttes et une plus faible $(0,28 \mathrm{~m})$ sur les billons.

L'analyse de variance montre que la hauteur des plants ne varie pas significativement suivant les traitements $(p=0,683)$ mais varie significativement suivant le type de sol $(\mathrm{p}=0)$. Le test d'additivité de Tukey renseigne qu'aucune interaction n'est observée entre le type de sol et le traitement pour le paramètre hauteur $(\mathrm{p}=0,961)$. La détermination des différences existant entre les types de sol sur la base du test de StudentNewman-Keuls montre que trois groupes de hauteurs se distinguaient suivant le type de sol : le groupe du sol vertisol argileux, le groupe du vertisol argilocalcaire superficiel et le groupe constitué des sols argileux sableux et des vertisols argilo-calcaires.

\section{Circonférence moyenne des régénérations naturelles}

Il ressort des résultats qu'au niveau du traitement témoin, la circonférence au collet des plants varie de $3,42 \mathrm{~cm}$ à $6,26 \mathrm{~cm}$ dans les interlignes et $2,42 \mathrm{~cm}$ à $6,19 \mathrm{~cm}$ sur les lignes avec la plus forte grosseur sur le vertisol argileux $(6,26 \mathrm{~cm})$ et la plus faible sur le sol argileux sableux $(2,42$ $\mathrm{cm})$. Sur les billons, la circonférence au collet enregistrée a varié de $3,69 \mathrm{~cm}$ à $7,06 \mathrm{~cm}$ dans les interlignes et de $2,74 \mathrm{~cm}$ à $6,23 \mathrm{~cm}$ sur les lignes avec la 
plus forte grosseur sur le vertisol argileux $(7,06 \mathrm{~cm})$ et la faible sur le sol argileux sableux $(2,74 \mathrm{~cm})$. Sur les buttes, elle pourrait varier de $3,01 \mathrm{~cm}$ à $6,54 \mathrm{~cm}$ dans les interlignes et de $2,17 \mathrm{~cm}$ à $6,62 \mathrm{~cm}$ sur les lignes avec la plus forte grosseur sur le vertisol argileux calcaire à faible profondeur $(6,62 \mathrm{~cm})$ et la plus faible sur le sol argileux sableux $(2,17 \mathrm{~cm})$.

Par rapport au facteur sol, sur le sol argileux sableux, la circonférence au collet des plants varie de $2,42 \mathrm{~cm}$ à $5,5 \mathrm{~cm}$ dans les interlignes et de 2,17 $\mathrm{cm}$ à $2,74 \mathrm{~cm}$ sur les lignes avec une forte grosseur sur le traitement des billons $(5,5 \mathrm{~cm})$ et une faible grosseur sur le traitement sur celui des buttes $(2,17 \mathrm{~cm})$. Sur le vertisol argileux, elle varie de $6,26 \mathrm{~cm}$ à 7,06 cm dans les interlignes et de $3,96 \mathrm{~cm}$ à $6,23 \mathrm{~cm}$ sur les lignes avec la plus forte grosseur sur les traitements des billons $(7,06 \mathrm{~cm})$ et la plus faible au niveau des buttes $(3,96$ $\mathrm{cm})$. Sur le vertisol argileux calcaire, circonférence au collet varie de 3,96 cm à 4,42 cm dans les interlignes et de $3,26 \mathrm{~cm}$ à 4,61 cm sur les lignes avec la plus forte grosseur $(4,61 \mathrm{~cm})$ sur le traitement butte et la plus faible $(3,26 \mathrm{~cm})$ au niveau des billons. Sur le vertisol argileux calcaire à faible profondeur, elle varie de $3,69 \mathrm{~cm}$ à $6,5 \mathrm{~cm}$ dans les interlignes et de $3,47 \mathrm{~cm} 6,62 \mathrm{~cm}$ au niveau des lignes avec la plus forte grosseur sur les buttes $(6,62 \mathrm{~cm})$ et la plus faible $(3,47 \mathrm{~cm})$ sur les billons.

L'analyse de variance montre que la circonférence des plants ne varie pas significativement suivant les traitements $(p=0,942)$ mais varie significativement suivant le type de sol $(\mathrm{p}=0)$. Le test d'additivité de Tukey renseigne qu'aucune interaction n'est observée entre le type de sol et le traitement pour le paramètre circonférence $(\mathrm{p}=0,530)$. La détermination des différences existant entre les types de sol sur la base du test de StudentNewman-Keuls montre que quatre groupes de circonférences se distinguent suivant le type de sol: le groupe du sol vertisol argileux, le groupe du vertisol argilo-calcaire et le groupe constitué des sols argileux sableux. Les vertisols argilo-calcaires superficiels ne se différencient pas statistiquement du sol vertisol argileux ni du vertisol argilo-calcaire.

\section{Comparaison des paramètres dendrométriques entre interligne et ligne}

Le test de student utilisé pour mettre en évidence les différences entre les interlignes et les lignes montre que pour l'ensemble des dispositifs réunis, la hauteur $(\mathrm{p}=0,096)$ et la circonférence des plants $(\mathrm{p}=0,07)$ ne varient pas significativement entre les interlignes et les lignes. Seul le nombre de plants varie significativement $(\mathrm{p}=0,021)$ entre interlignes et lignes.

\section{Evaluation des coûts d'investissement en fonction des traitements.}

Les coûts des travaux, hors abattage et dégagement des rémanents sont présentés dans le tableau 11. Il ressort que le buttage ou le billonnage sont trois 
fois plus onéreux que le traitement témoin pour lequel il n'y a aucun apport de graines autres que celles venant du peuplement abattu.

Tableau 11 : Point des dépenses réalisées par traitement.

\begin{tabular}{crrr}
\hline \multirow{2}{*}{ Activités } & \multicolumn{3}{c}{ Traitements (FCFA CFA/ha) } \\
\cline { 2 - 4 } & \multicolumn{1}{c}{ Témoin } & \multicolumn{1}{c}{ Billons } & \multicolumn{1}{c}{ Buttes } \\
\hline Réalisations des buttes et billons & 0 & 186667 & 180000 \\
\hline \multirow{2}{*}{ Achat des graines de teck (2 sacs/ha de 40kg) } & 0 & 24000 & 24000 \\
\hline Epandage des graines de teck & 0 & 2520 & 2520 \\
\hline Entretien manuel (5 entretiens) & 90000 & 90000 & 90000 \\
\hline Maintenance des ouvriers (10\%) & 9000 & 30319 & 29652 \\
\hline TOTAL & 99000 & 333506 & 326172 \\
\hline
\end{tabular}

\section{Discussion}

\section{Effets des traitements et du type de sol}

Le teck (Tectona grandis L. f) présente un potentiel très élevé de régénération naturelle sur les vertisols hydromorphes de la Lama tout comme cela avait déjà été démontré depuis les années 1990 pour les sols ferralitiques et les sols ferrugineux du Bénin (Lutz, 1991; Akouèhou \& Ayélo, 2005). Forget (1988) affirme que l'hydromorphie favorise une meilleure survie de la régénération, en particulier au cours de la saison sèche et qu'une moindre mortalité et une croissance légèrement supérieure favorisent une plus forte densité des arbres aux stades juvéniles et gaulis.

La densité, la hauteur et la circonférence au collet des plants diffèrent significativement selon les types de sol et confirment ainsi notre première hypothèse quant à l'importance du sol pour la régénération naturelle assistée (RNA). D'autres auteurs ont également mis en évidence le rôle déterminant du sol dans l'établissement de la régénération des ligneux (Ouédraogo et al., 2006 ; Ky-Dembele et al., 2007 ; Avocevou-Ayisso et al., 2009 ; Schumann et al., 2011 ; Nacoulma et al., 2011 ; Bondé et al., 2013 ; Assédé et al, 2015). Sur le vertisol argileux les régénérations naturelles ont été abondantes (61 578 plants/ha) et ont montré une bonne croissance en hauteur $(0,73 \mathrm{~m})$ et en circonférence au collet $(7,06 \mathrm{~cm})$. À l'opposé, sur les sols argileux à recouvrement sableux, les croissances ont été faibles tant en hauteur $(0,15 \mathrm{~m})$ qu'en circonférence au collet $(2,17 \mathrm{~cm})$, ce que Lepoutre (1965) avait déjà observé en notant que plus la couche sableuse est épaisse, plus les racines des semis tardent à atteindre la couche d'argile où elles pourront puiser l'eau nécessaire à leur survie et à leur résistance. Les régénérations les moins denses (8 257 plants/ha) ont été observées sur les vertisols argilo-sableux superficiels, ce qui pourrait s'expliquer en partie par une plus faible densité des semenciers sur ce type de sol. Sinsin et al. (2004) et de Biaou et al. (2011) affirment aussi que le potentiel de régénération est favorisé par une densité optimale de 
semenciers alors que Boudy (1948) précise que la régénération par semis est aléatoire surtout quand les conditions écologiques de base sont perturbées.

$\mathrm{Au}$ niveau des circonférences au collet, quatre groupes se distinguent suivant le type de sol: le groupe (a) du vertisol argileux, le groupe (b) du vertisol argileux calcaire et le groupe (c) constitué des sols argileux sableux. Les vertisols argilo-calcaire superficiels peuvent être classées dans le groupe (ab) du vertisol argileux ou dans le groupe du vertisol argileux calcaire. Le groupe (c) constitué ici peut être dû à la provenance. Sur le sol AS la provenance du teck est locale alors qu'elle est tanzanienne pour tous les autres. Cela peut expliquer la circonférence moyenne relativement basse obtenue au niveau de ce sol. On pourra donc considérer deux groupes: AS d'une part, et VA, VAC, VACS d'autre part. Le second groupe peut se subdiviser en deux sous-groupes: VAC, VACS puis VA.

La densité, la hauteur et la circonférence au collet des plants ne varient pas significativement suivant le type de travail du sol, ce qui infirme notre seconde hypothèse sur l'importance du travail du sol sur la régénération naturelle. Aucune interaction n'est aussi observée entre le type de sol et le travail de celui-ci. Cependant, de façon générale, les densités observées au niveau des lignes où le sol a été travaillé dépassent celles des interlignes non travaillées. Par contre, les hauteurs et les circonférences des plants des interlignes sont supérieures à celles des plants sur les lignes, ce qui pourrait s'expliquer par une plus forte densité de semis sur les lignes travaillées entrainant une concurrence pour l'eau et les éléments nutritifs. Akouèhou \& Ayélo (2005) ont ainsi montré que la densité des régénérations de teck de moins de trois ans influence négativement la croissance en hauteur et en circonférence.

\section{Coûts d'investissements et de rentabilité des traitements}

Les coûts des travaux destinés à favoriser l'installation des semis se sont limités aux désherbages pour le «témoin » (99.000 francs CFA/ha) soit nettement moins que le billonnage (333.506 francs CFA/ha) ou le façonnage de buttes (326.172 francs CFA/ha) associés à des apports de graines supplémentaires. Compte tenu de ces différences de coûts, et des faibles différences constatées sur la densité des semis ainsi que sur leur croissance, la technique témoin peut être recommandée pour régénérer, après exploitation, les teckeraies sur les vertisols et les sols hydromorphes de la Lama.

Il faut toutefois noter qu'associer les populations riveraines aux activités de nettoyage et de dégagement des rémanents a permis de diminuer considérablement les coûts des travaux de terrains. Les populations étant rémunérées par le bois qu'elles récupèrent pour la vente ou leur usage personnel. L'apport des riverains apparaît indispensable pour la réussite des régénérations naturelles à moindre coût. Cependant, il est indispensable 
d'exploiter le peuplement et de procéder au dégagement et au nettoyage des parterres de coupe avant la période pluvieuse. Ce travail de dégagement et de nettoyage passe inévitablement par la sensibilisation et l'adhésion des populations riveraines des plantations de la Lama aux activités de l'exploitation forestières.

\section{Conclusion}

La maîtrise des techniques de régénération naturelle assistée constitue aujourd'hui plus que jamais, une priorité pour les gestionnaires des plantations de Tectona grandis L.f. Les sols hydromorphes et les vertisols ne constituent pas un obstacle majeur à l'installation de régénérations naturelles. La densité, la hauteur et la circonférence au collet des régénérations obtenues ne varient pas significativement en fonction du travail préparatoire du sol mais selon le type de sol sans qu'il y ait d'interaction entre ces deux facteurs.

Le coût d'installation du témoin, qui consiste en l'enlèvement des rémanents après l'exploitation de la parcelle suivi de désherbages, est le moins onéreux. Cette technique apparaît donc comme la plus appropriée pour le renouvellement de ces plantations de teck. Cette méthode de régénération doit cependant respecter strictement les conditions et les critères de sa mise en œuvre, c'est à dire exploiter le peuplement à blanc en saison sèche et dégager les rémanents issus des houppiers, faucher les herbacées et nettoyer le parterre de coupe avant la saison pluvieuse. Il est donc indispensable de mobiliser tous les moyens pour réaliser les coupes, le dégagement et le nettoyage à temps. Ce n'est qu'à ces conditions que la régénération naturelle assistée donnera les résultats attendus.

Pour minimiser les coûts en mobilisant les populations riveraines, il est nécessaire pour les gestionnaires forestiers de sensibiliser davantage les populations riveraines qui tireront un bénéfice de la récupération des rémanents.

Les résultats obtenus ne concernent pas les bas-fonds qui peuvent être temporairement inondés. En effet, l'hydromorphie de ces vertisols et le dérèglement climatique observé ces dernières années peuvent engendrer l'échec des régénérations naturelles tant souhaitées. Dans ces zones sensibles nous suggérons de faire un billonnage suivi d'un semis de graines sur les billons pour éviter que les plantules ne soient asphyxiées en cas d'inondation. Par la suite, cela ne posera plus de problème car le teck, une fois bien installé, supporte une submersion si elle ne dure pas trop longtemps. 


\section{References:}

1. Akouèhou S. G. \& Ayelo H. G. (2005). De la conduite des peuplements issus de la régénération naturelle à la sylviculture : cas du teck (Tectona grandis) dans de la plantation domaniale de Djigbé au sud Bénin. Bulletin de la Recherche Agronomique du Bénin, 47 : 7-18.

2. Akpene A. D., Chaix G., Monteuuis O., Langbour P., Guibal D., Tomazello M. F., Kokutse A. D., Kokou K (2014). Mise au point d'une stratégie d'amélioration des plantations de teck au Togo. Conférence Matériaux 2014 - Colloque Ecomatériau, Nov 2014, Montpellier, France. Collection ECOMATERIAU. <hal-01144499>

3. Assédé E. S. P., Azihou F. A., Adomou A. C., Oumorou M. \& Sinsin B. (2015). Effet du relief sur la régénération des espèces ligneuses en zone soudanienne du Bénin. Bois et Forêts des Tropiques, 326(4): 15.

4. Avocevou-Ayisso C., Sinsin B., Adégbidi A., Dossou G. \& Van Damme P. (2009). Sustainable use of non-timber forest products: Impact of fruit harvesting on Pentadesma butyracea regeneration and financial analysis of its products trade in Benin. Forest Ecology and Management, 257: 1930-1938.

5. Béhaghel I. (1999). Etat des plantations de teck (Tectona grandis 1.f.) dans le monde. [The state of teak (Tectona grandis 1.f.) plantations in the world; Estado de las plantaciones de teca (Tectona grandis 1.f.) en el mundo]. [archive]. Bois et forêts des tropiques, 262: 6-18.

6. Biaou S. S. H., Holmgren M., Sterck F. J. \& Mohren G. M. J. (2011). Stress-Driven Changes in the Strength of Facilitation on Tree Seedling Establishment in West African Woodlands. Biotropica, 43(1): 23-30.

7. Bondé L., Ouédraogo O., Kagembèga F. \& Boussim J. I. (2013). Impact des gradients topographique et anthropique sur la diversité des formations ligneuses soudaniennes. Bois et Forêts des Tropiques, 318(318) : 15-25.

8. Boudy P. (1948). Economie forestière Nord-Africaine. Milieu physique et humain. Ed. Larose, Paris, Tome I, 684 p.

9. FAO (2005). Evaluation des Ressources Forestières Mondiales 2005. FAO. Rome Italie. 320 p.

10. FAO (2010). Evaluation des ressources forestières mondiales 2010 Rapport principal. Rome Italie, $317 \mathrm{p}$.

11. FAO (2014). Situation des forêts du monde : Mieux tirer parti des avantages socioéconomiques des forêts. Rome Italie. 146 p.

12. FAO (2016). Situation des Forêts du monde 2016 (SOFO). Forêts et agriculture : défis et possibilités concernant l'utilisation des terres. Rome Italie. 138 p. 
13. Forget P. M. (1988). Dissémination et régénération naturelle de huit espèces d'arbres en forêt guyanaise. Thèse de Doctorat, Université Paris VI, $245 \mathrm{p}$

14. Gnanguènon-Guessè D., Kakpo S. B., Aoudji A. K. N., Koura K. \& Ganglo J. C. (2015). Exploitation forestière à faible impact dans les teckeraies de la Lama (Benin, Afrique de l'ouest). Science de la vie, de la terre et agronomie, 03(02): 37-44.

15. Kakpo S. B., Yêhouénou Tessi D. R., Gbètoho J. A., Aoudji A. K. N. \& Ganglo J. C. (2018). Répartition spatiale de Cola millenii K. Schum., Dialium guineense Wild. et Afzelia africana Smith ex Pers. dans les forêts secondaires du Sud Benin (Afrique de l'Ouest). International Journal of Biological and Chemical Sciences, 12(1): 353362. Doi: https://dx.doi.org/10.4314/ijbcs.v12i1.28

16. Kjaer E. D., Kaosa-ard A. \& Suangtho V. (2000). Domestication of teak through tree improvement. Options, possible gains and critical factors. In: Site, technology and productivity of teak plantations. FORSPA Publication $\mathrm{N}^{\circ}$ 24/2000, TEAKNET Publication $\mathrm{N}^{\mathrm{o}} 3$ : $161-$ $189 \mathrm{p}$.

17. Ky-Dembele C., Tigabu M., Bayala J., Ouédraogo S. J. \& Odén P. C. (2007). The relative importance of different regeneration mechanisms in a selectively cut savanna-woodland in Burkina Faso, West Africa. Forest Ecology and Management, 243(1): 28-38.

18. Lepoutre B., (1965). Régénération artificielle du chêne-liège et équilibre climacique de la subéraie en forêt de la Mamora. Ann. Rech. Forest. Rabat, 9: 1-86 p.

19. Louppe, D. (2005). Tectona grandis L.f. [Internet] Fiche de Protabase. Louppe, D. et Oteng-Amoako, A. (Editeurs). PROTA (Plant Resources of Tropical Africa / Ressources végétales de l'Afrique tropicale), Wageningen, Pays Bas. <http://database.prota.org/recherche.htm>. Visité le 1 février 2008.

20. Lutz W. (1991). La régénération naturelle de Tectona grandis dans les plantations des secteurs d'Agrimey, Djigbé et Toffo au Sud du Bénin. GTZ, 68 p.

21. Marien J. N. \& Mallet B. (2004). Nouvelles perspectives pour les plantations forestières en Afrique centrale. Bois et forêts des tropiques, 282(4): 67-79.

22. Monteuuis O. \& Maître H. F. (2007). New developments in teak cloning lead to better plantation stock. ITTO Tropical Forest Update $17 / 3$.

23. Nacoulma B. M. I., Schumann K., Traoré S., Bernhardt-Römermann M., Hahn K., Wittig R. \& Thiombiano A. (2011). Impacts of land-use on West African savanna vegetation: a comparison between protected 
and communal area in Burkina Faso. Biodiversity and Conservation, 20(14): 3341-3362.

24. Ouédraogo A., Thiombiano A., Hahn-Hadjali K. \& Guinko S. (2006). Structure du peuplement juvénile et potentialités de régénération des ligneux dans l'Est du Burkina Faso. Études de la Flore et de la Végétation du Burkina Faso, 10: 17-24 .

25. Putz F. E. \& Redford K. H. (2010). The Importance of Defining 'Forest': Tropical Forest Degradation, Deforestation, Long-term Phase Shifts, and Further Transitions. Biotropica, 42(1): 10-20. Doi: 10.1111/j.1744-7429.2009.00567.

26. Schumann K., Wittig R., Thiombiano A., Becker U. \& Hahn K. (2011). Impact of land-use type and harvesting on population structure of a non-timber forest product-providing tree in a semi-arid savanna, West Africa. Biological Conservation, 144(9): 2369-2376.

27. Sinsin B., Eyog-Matig O., Assogbadjo A. E., Gaoué O. G. \& Sinadouwirou T. (2004). Dendrometric characteristics as indicators of pressure of Afzelia africana Sm. trees dynamics in different climatic zones of Benin. Biodiversity and Conservation, 13(8): 1555-1570.

28. Verhaegen D., Fofana I. J., Zénor A. L. \& Ofori D. (2010). What is the genetic origin of teak (Tectona grandis L.) introduced in Africa and in Indonesia? Tree Genetics \& Genomes, 6(7): 17-733. Doi 10.1007/s11295-010-0286-X

29. Viennot M. (1966). Etude des sols de la dépression de la Lama et de ses bordures. Carte pédologique de reconnaissance au 1/50000. ORSTOM, Cotonou, $58 \mathrm{p}$.

30. Akouèhou S. G. \& Ayelo H. G. (2005). De la conduite des peuplements issus de la régénération naturelle à la sylviculture : cas du teck (Tectona grandis) dans de la plantation domaniale de Djigbé au sud Bénin. Bulletin de la Recherche Agronomique du Bénin, 47 : 7-18.

31. Akpene A. D., Chaix G., Monteuuis O., Langbour P., Guibal D., Tomazello M. F., Kokutse A. D., Kokou K (2014). Mise au point d'une stratégie d'amélioration des plantations de teck au Togo. Conférence Matériaux 2014 - Colloque Ecomatériau, Nov 2014, Montpellier, France. Collection ECOMATERIAU. <hal-01144499>

32. Assédé E. S. P., Azihou F. A., Adomou A. C., Oumorou M. \& Sinsin B. (2015). Effet du relief sur la régénération des espèces ligneuses en zone soudanienne du Bénin. Bois et Forêts des Tropiques, 326(4): 15.

33. Avocevou-Ayisso C., Sinsin B., Adégbidi A., Dossou G. \& Van Damme P. (2009). Sustainable use of non-timber forest products: Impact of fruit harvesting on Pentadesma butyracea regeneration and financial analysis of its products trade in Benin. Forest Ecology and Management, 257: 1930-1938. 
34. Béhaghel I. (1999). Etat des plantations de teck (Tectona grandis 1.f.) dans le monde. [The state of teak (Tectona grandis 1.f.) plantations in the world; Estado de las plantaciones de teca (Tectona grandis 1.f.) en el mundo]. [archive]. Bois et forêts des tropiques, 262: 6-18.

35. Biaou S. S. H., Holmgren M., Sterck F. J. \& Mohren G. M. J. (2011). Stress-Driven Changes in the Strength of Facilitation on Tree Seedling Establishment in West African Woodlands. Biotropica, 43(1): 23-30.

36. Bondé L., Ouédraogo O., Kagembèga F. \& Boussim J. I. (2013). Impact des gradients topographique et anthropique sur la diversité des formations ligneuses soudaniennes. Bois et Forêts des Tropiques, 318(318) : 15-25.

37. Boudy P. (1948). Economie forestière Nord-Africaine. Milieu physique et humain. Ed. Larose, Paris, Tome I, 684 p.

38. FAO (2005). Evaluation des Ressources Forestières Mondiales 2005. FAO. Rome Italie. 320 p.

39. FAO (2010). Evaluation des ressources forestières mondiales 2010 Rapport principal. Rome Italie, $317 \mathrm{p}$.

40. FAO (2014). Situation des forêts du monde : Mieux tirer parti des avantages socioéconomiques des forêts. Rome Italie. 146 p.

41. FAO (2016). Situation des Forêts du monde 2016 (SOFO). Forêts et agriculture : défis et possibilités concernant l'utilisation des terres. Rome Italie. 138 p.

42. Forget P. M. (1988). Dissémination et régénération naturelle de huit espèces d'arbres en forêt guyanaise. Thèse de Doctorat, Université Paris VI, $245 \mathrm{p}$

43. Gnanguènon-Guessè D., Kakpo S. B., Aoudji A. K. N., Koura K. \& Ganglo J. C. (2015). Exploitation forestière à faible impact dans les teckeraies de la Lama (Benin, Afrique de l'ouest). Science de la vie, de la terre et agronomie, 03(02): 37-44.

44. Kakpo S. B., Yêhouénou Tessi D. R., Gbètoho J. A., Aoudji A. K. N. \& Ganglo J. C. (2018). Répartition spatiale de Cola millenii K. Schum., Dialium guineense Wild. et Afzelia africana Smith ex Pers. dans les forêts secondaires du Sud Benin (Afrique de l'Ouest). International Journal of Biological and Chemical Sciences, 12(1): 353362. Doi: https://dx.doi.org/10.4314/ijbcs.v12i1.28

45. Kjaer E. D., Kaosa-ard A. \& Suangtho V. (2000). Domestication of teak through tree improvement. Options, possible gains and critical factors. In: Site, technology and productivity of teak plantations. FORSPA Publication $N^{0} 24 / 2000$, TEAKNET Publication $\mathrm{N}^{\circ} 3$ : $161-$ $189 \mathrm{p}$.

46. Ky-Dembele C., Tigabu M., Bayala J., Ouédraogo S. J. \& Odén P. C. (2007). The relative importance of different regeneration mechanisms 
in a selectively cut savanna-woodland in Burkina Faso, West Africa. Forest Ecology and Management, 243(1): 28-38.

47. Lepoutre B., (1965). Régénération artificielle du chêne-liège et équilibre climacique de la subéraie en forêt de la Mamora. Ann. Rech. Forest. Rabat, 9: 1-86 p.

48. Louppe, D. (2005). Tectona grandis L.f. [Internet] Fiche de Protabase. Louppe, D. et Oteng-Amoako, A. (Editeurs). PROTA (Plant Resources of Tropical Africa / Ressources végétales de l'Afrique tropicale), Wageningen, Pays Bas. <http://database.prota.org/recherche.htm>. Visité le 1 février 2008.

49. Lutz W. (1991). La régénération naturelle de Tectona grandis dans les plantations des secteurs d'Agrimey, Djigbé et Toffo au Sud du Bénin. GTZ, 68 p.

50. Marien J. N. \& Mallet B. (2004). Nouvelles perspectives pour les plantations forestières en Afrique centrale. Bois et forêts des tropiques, 282(4): 67-79.

51. Monteuuis O. \& Maître H. F. (2007). New developments in teak cloning lead to better plantation stock. ITTO Tropical Forest Update $17 / 3$.

52. Nacoulma B. M. I., Schumann K., Traoré S., Bernhardt-Römermann M., Hahn K., Wittig R. \& Thiombiano A. (2011). Impacts of land-use on West African savanna vegetation: a comparison between protected and communal area in Burkina Faso. Biodiversity and Conservation, 20(14): 3341-3362.

53. Ouédraogo A., Thiombiano A., Hahn-Hadjali K. \& Guinko S. (2006). Structure du peuplement juvénile et potentialités de régénération des ligneux dans l'Est du Burkina Faso. Études de la Flore et de la Végétation du Burkina Faso, 10: 17-24 .

54. Putz F. E. \& Redford K. H. (2010). The Importance of Defining 'Forest': Tropical Forest Degradation, Deforestation, Long-term Phase Shifts, and Further Transitions. Biotropica, 42(1): 10-20. Doi: 10.1111/j.1744-7429.2009.00567.

55. Schumann K., Wittig R., Thiombiano A., Becker U. \& Hahn K. (2011). Impact of land-use type and harvesting on population structure of a non-timber forest product-providing tree in a semi-arid savanna, West Africa. Biological Conservation, 144(9): 2369-2376.

56. Sinsin B., Eyog-Matig O., Assogbadjo A. E., Gaoué O. G. \& Sinadouwirou T. (2004). Dendrometric characteristics as indicators of pressure of Afzelia africana $\mathrm{Sm}$. trees dynamics in different climatic zones of Benin. Biodiversity and Conservation, 13(8): 1555-1570.

57. Verhaegen D., Fofana I. J., Zénor A. L. \& Ofori D. (2010). What is the genetic origin of teak (Tectona grandis L.) introduced in Africa and in 
Indonesia? Tree Genetics \& Genomes, 6(7): 17-733. Doi 10.1007/s11295-010-0286-X

58. Viennot M. (1966). Etude des sols de la dépression de la Lama et de ses bordures. Carte pédologique de reconnaissance au 1/50000. ORSTOM, Cotonou, $58 \mathrm{p}$. 